\title{
Modeling crosstalk and afterpulsing in silicon photomultipliers
}

\author{
J. Rosado ${ }^{\mathrm{a}, *}$, V.M. Aranda ${ }^{\mathrm{a}}$, F. Blanco ${ }^{\mathrm{a}}, \mathrm{F}$. Arqueros $^{\mathrm{a}}$ \\ ${ }^{a}$ Departamento de Física Atómica, Molecular y Nuclear, Facultad de Ciencias Físicas, \\ Universidad Complutense de Madrid, E-28040 Madrid, Spain
}

\begin{abstract}
An experimental method to characterize the crosstalk and afterpulsing in silicon photomultipliers has been developed and applied to two detectors fabricated by Hamamatsu. An analytical model of optical crosstalk that we presented in a previous publication has been compared with new measurements, confirming our results. Progresses on a statistical model to describe afterpulsing and delayed crosstalk are also shown and compared with preliminary experimental data.
\end{abstract}

Keywords: Silicon photomultipliers, crosstalk, afterpulsing

\section{Introduction}

Silicon photomultipliers (SiPM) have excellent time and photon counting resolutions, among other qualities. However, crosstalk and afterpulsing seriously limit the performance of these photon detectors. These phenomena are the production of parasitic avalanches in either the pixel where a primary avalanche was triggered (afterpulsing) or a neighboring one (crosstalk). Their main effects are to increase both the count rate and the total charge collected for an input light pulse, e.g., from a scintillator.

The physical processes considered in our analysis are illustrated in figure 1. In the first place, a charge carrier of a primary avalanche can be trapped in a crystal defect within the pixel, then when the avalanche has already been quenched, the carrier may be released triggering a new avalanche. We

\footnotetext{
${ }^{*}$ Corresponding author

Email address: jaime_ros@fis.ucm.es (J. Rosado)
} 

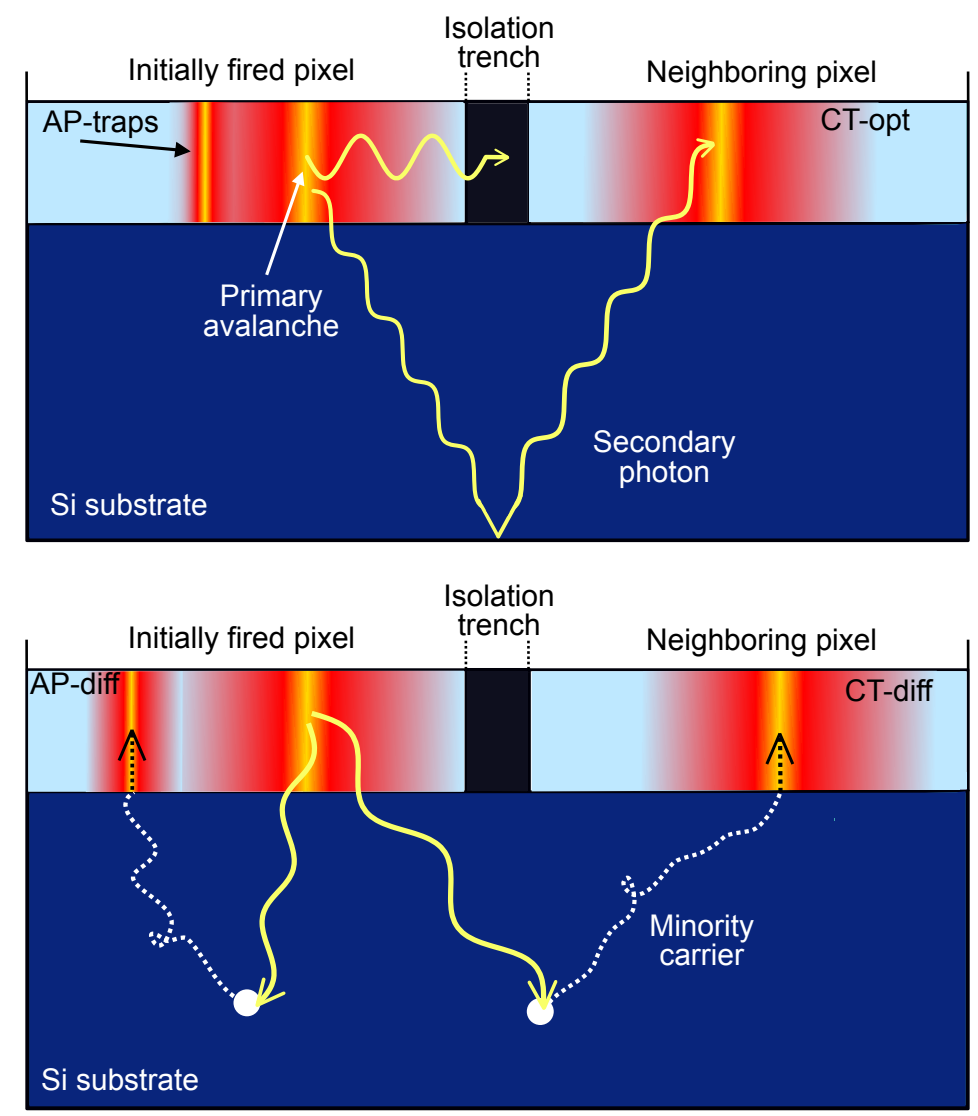

Figure 1: Physical processes considered in the analysis. Top: Afterpulsing due to carriers trapped in crystal defects within a pixel, and almost instantaneous optical crosstalk due to secondary photons reaching neighboring pixels. Bottom: Secondary photons generate minority carriers in the silicon substrate that may diffuse back to either the primary pixel or a neighboring one, inducing respectively afterpulsing or delayed crosstalk.

refer to this process as afterpulsing due to traps (AP-traps) and it has as many components as types of traps in the silicon lattice. In the second place, secondary photons of the primary avalanche may reach neighboring pixels triggering almost simultaneously other avalanches. This is the socalled optical crosstalk (CT-opt). Some SiPMs incorporate trenches filled with an optical absorber surrounding pixels to prevent this effect, but CTopt can still be produced by photons reflected on the bottom surface of the detector. Finally, minority carriers generated in the silicon substrate by secondary photons of the primary avalanche can diffuse back to either the 
primary pixel or to a neighboring one, contributing respectively to crosstalk (CT-diff) or afterpulsing (AP-diff) $]^{1}$ The CT-diff is usually referred to as delayed crosstalk because of the relatively long diffusion times, in contrast to the CT-opt, which is also called prompt crosstalk.

We presented in [1] an experimental method and an analytical model to describe the CT-opt in a SiPM fabricated by Hamamatsu. In the present work, our study has been extended with new measurements for the same SiPM as well as for a second SiPM, also from Hamamatsu. In addition, we have constructed a statistical model accounting for AP-traps, CT-diff and AP-diff, and preliminary measurements to validate it are shown. A similar analysis of afterpulsing and CT-diff in a STMicroelectronics SiPM has recently been presented in [2].

\section{Experimental method}

The experimental method has been described in detail in [1], and only an overview is given here. The setup consists of the SiPM with the associated bias circuit, a fast amplifier, and a digital oscilloscope to register and store the signals for later analysis. Two SiPMs from Hamamatsu were tested: the S10362-11-100C (the one previously used in [1]) and the S10362-33-100C. Both detectors have a pixel pitch of $100 \mu \mathrm{m}$ and basically the same characteristics, but the first SiPM has 100 pixels while the second one has 900 pixels. Measurements were performed at dark conditions, that is, an only primary pixel is expected to be triggered at a time. So far, we have characterized the intrinsic crosstalk and afterpulsing (i.e., no scintillator was attached to the detector) at room temperature.

An algorithm has been developed to perform a detailed waveform analysis of the recorded signals. This dedicated software calculates numerically the deconvolution of the signal with a negative exponential function that describes the decay of pulses (time constant of $23 \mathrm{~ns}$ for both detectors). This way, pulses are identified as distinct peaks in the deconvolved signal even if they overlap in the original signal. The position and height of the deconvolution peak provide us relative measures of the arrival time and amplitude of each pulse. The algorithm resolves and measures the parameters of pulses as close as 6 ns. In addition, events with two or more pulses with smaller time

\footnotetext{
${ }^{1}$ Only the minority carriers are accelerated by the electric field and so able to trigger an avalanche when they reach a pixel.
} 
differences (down to $\sim 1 \mathrm{~ns}$ ) are identified by analyzing the shape and width at threshold level of the deconvolved signal. This allowed us to characterize separately the nearly instantaneous CT-opt and the other phenomena that result in delayed secondary pulses. A more precise measurement of the pulse amplitude is obtained as the pulse height with baseline substraction. To do that, the software performs an exponential extrapolation of the original signal in a small region prior to each pulse.

\section{Optical crosstalk}

The CT-opt gives rise to pulses with amplitudes two or more times higher than that corresponding to only one triggered pixel. The relative intensities of the pulse amplitude spectrum measured at dark conditions thus provides the probability distribution $P(k)$ of the total number $k$ of simultaneously triggered pixels per primary avalanche, i.e., $k-1$ CT-opt excitations. We produced this spectrum using the pulse height with baseline substraction to optimize the resolution in $k$. In addition, quality cuts were applied to reject low-amplitude pulses due to pixels that were triggered while being recharged after the last avalanche breakdown.

We presented in [1] a statistical model based on the assumption that secondary photons can only reach a certain neighborhood of pixels around the primary one. Cascades of CT-opt excitations propagating through the whole array of pixels were included, but taking into account that pixels that have already been triggered cannot be triggered twice. The $P(k)$ distribution was then calculated by identifying all the CT-opt "histories" that contribute to each probability considering four possible arrangements of neighbors: the 4 nearest neighbors, the 8 nearest neighbors, the 8 L-connected neighbors and all neighbors (see [1] for details). For simplicity, we assumed the same CTopt probability for any neighbor and ignored the boundaries of the array of pixels for the calculations. Analytical expressions for the mean and variance of the $P(k)$ distribution were also obtained.

In figure 2, the model predictions for the four arrangements of neighbors are compared with the experimental probabilities obtained at a given bias voltage for both the S10362-11-100C detector (top plot, taken from [1]) and the S10362-33-100C detector (bottom plot). In particular, the probability ratio (theoretical over experimental) is represented versus $k$ from 1 to 5 , where the model predictions were normalized so that the first probability $P(1)$ matches the experimental one, i.e., their ratio is unity. The error bars 

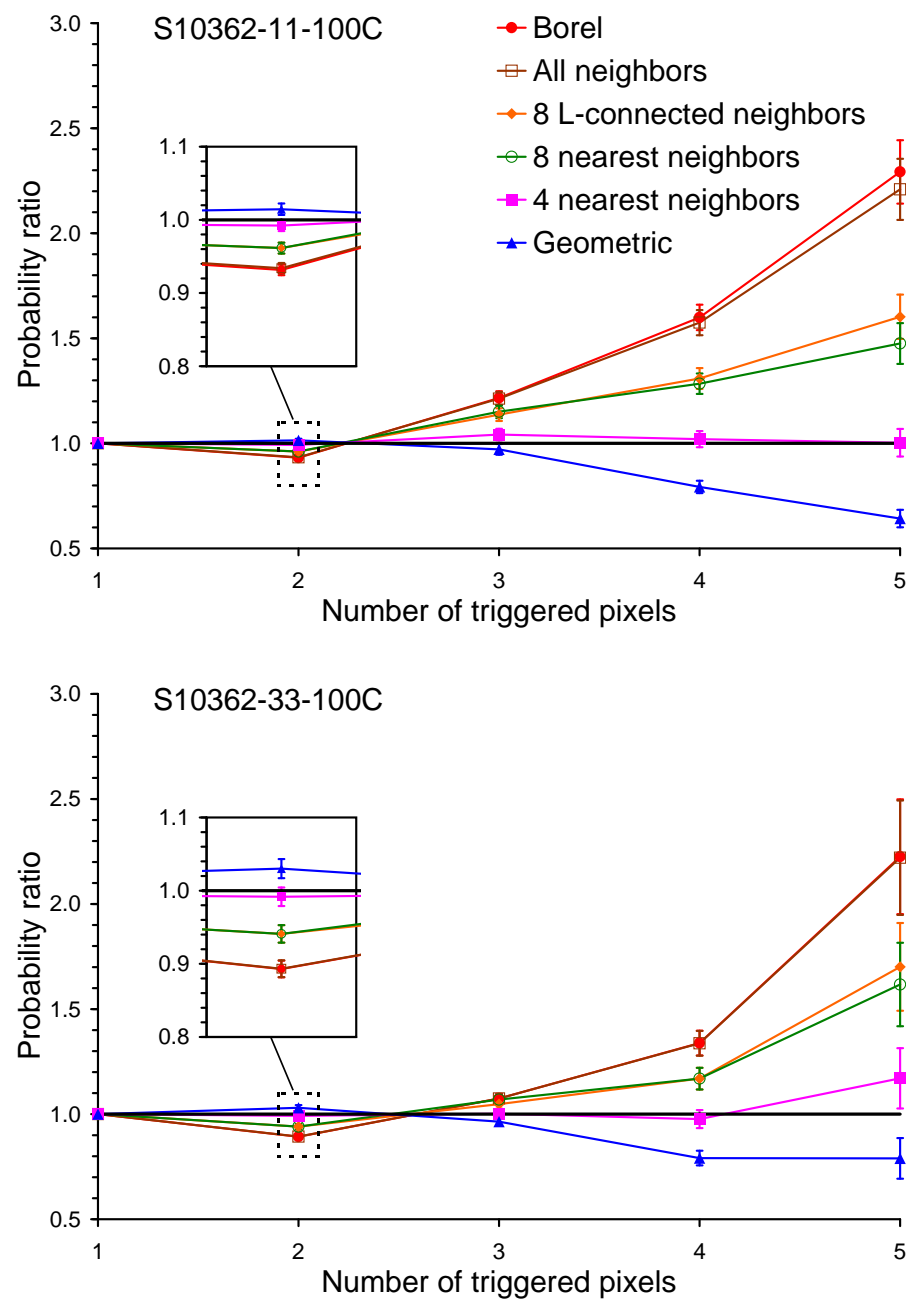

Figure 2: Ratio of model predictions of $P(k)$ for optical crosstalk over the experimental ones measured at dark conditions and a given bias voltage. Results for the two tested detectors are consistent with the hypothesis of the 4 nearest neighbors. See text for details.

of the remaining ratios were determined propagating the uncertainties of the experimental probabilities. The comparison includes two previous analytical models described in [3], where $P(k)$ is assumed to follow a geometric or a Borel distribution. As explained in [1], these two models can be regarded as limit situations of ours. 

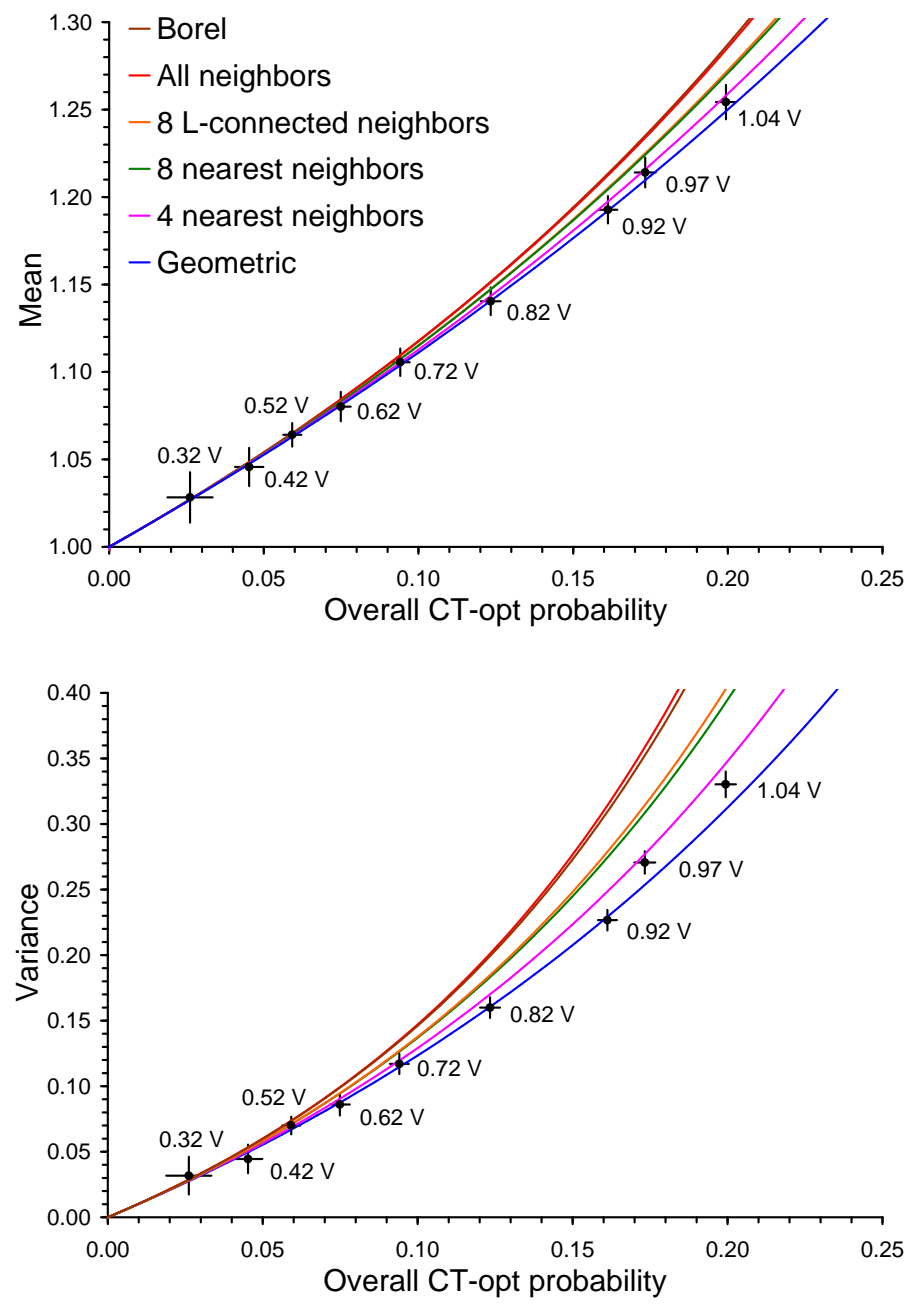

Figure 3: Comparison of the model predictions (lines) with experimental data (points) of optical crosstalk taken at different bias voltages. Results are for the mean (top) and the variance (bottom) of the $P(k)$ distribution for the S10362-11-100C detector. The legend shows the order from top to down of the lines representing the model predictions.

In figure 3, the model predictions of the mean and variance of the $P(k)$ distribution are compared with new experimental results for the S10362-11$100 \mathrm{C}$ detector as a function of the overall CT-opt probability, defined as $1-P(1)$. The overvoltage, i.e., bias voltage minus breakdown voltage, at which measurements were performed is also indicated for each data point 
(the overall CT-opt probability grows quadratically with overvoltage).

The comparisons shown in figures 2 and 3 reveal that the only model consistent with our experimental data is the one assuming that CT-opt solely takes place in the 4 nearest neighbors of each pixel. This is the case for both tested detectors, which have different array sizes. Therefore, it can also be concluded that border effects are unimportant to describe CT-opt effects at these conditions.

\section{Afterpulsing and delayed crosstalk}

At dark conditions, the distribution of the pulse arrival time measured with respect to the previous pulse is a combination of the contributions of both uncorrelated dark noise and correlated secondary pulses (i.e., AP-traps, AP-diff and CT-diff). Since we were interested in measuring the probability of secondary pulses per primary avalanche, we obtained this time distribution selecting pairs of pulses in which the first one (the primary) has an amplitude corresponding to one only triggered pixel, that is, without CT-opt. In addition, this first pulse of the pair was required to be at least $500 \mathrm{~ns}$ far from previous pulses to avoid mixing contributions of secondary pulses from different primaries.

Assuming that the total number of secondary pulses of each type per primary avalanche is Poisson distributed, the above time distribution can be expressed as

$$
\begin{aligned}
p(t) \cdot \mathrm{d} t= & \exp \left[-R_{\mathrm{DC}} \cdot\left(t-t_{\text {min }}\right)-\sum_{i} \lambda_{i} \cdot \int_{t_{\text {min }}}^{t} f_{i}(s) \cdot \mathrm{d} s\right] \\
& {\left[R_{\mathrm{DC}}+\sum_{i} \lambda_{i} \cdot f_{i}(t)\right] \cdot \mathrm{d} t, }
\end{aligned}
$$

where $t_{\min }=10 \mathrm{~ns}$ is the minimum time difference allowed between pulses due to analysis limitations, $R_{\mathrm{DC}}$ is the dark count rate, $\lambda_{i}$ is the mean number of secondary pulses of type $i$ per primary avalanche, and $f_{i}(t)$ is the corresponding normalized time distribution. The details of this model, in particular the parameterization of the $f_{i}(t)$ functions, are still under progress and will be explained in a paper in preparation. Only the general aspects are given below. 
The $f(t)$ distribution for AP-traps was characterized by the product of three factors: a negative exponential distribution describing the release of trapped carriers; the exponential pixel recharging after the primary avalanche, which is proportional to both the gain and the probability of the secondary avalanche; and the $t$-dependent fraction of afterpulses (of any type) with amplitudes above the detection threshold applied in the analysis software. The last two factors, which are also applicable to the $f(t)$ distribution for AP-diff, account for the steep break of the experimental time distribution between 20 and 30 ns (see figure 4) and were determined from the time-amplitude information of afterpulses with short delay ${ }^{2}$

A Monte Carlo simulation was carried out to describe the timing and relative contributions of the AP-diff and CT-diff effects (bottom plot of figure 1). Values for the physical parameters related to the emission and absorption of secondary photons, the diffusion of minority carriers and the layer configuration of the chip were varied within a wide interval around typical data taken from the literature (e.g., see [4]). Preliminary simulation results showed that the transient time distribution can be properly described by the product of the exponential decay of the number of minority carriers due to recombination mechanisms and a negative power law. Also, the relative contributions of AP-diff and CT-diff were found to be strongly dependent on the pixel pitch. For instance, for a very small pixel pitch of $10 \mu \mathrm{m}$, the AP-diff component is negligible compared to the CT-diff one, while both components are significant for a pixel pitch of $100 \mu \mathrm{m}$ (present case).

A fit of our model to experimental data for the S10362-11-100C detector at an overvoltage of $\sim 1 \mathrm{~V}$ is shown in figure 4, where the contributions of the different types of secondary pulses and of dark counts are represented by thin lines. The corresponding accumulated contributions are also shown in the inset of the figure in a linear scale. This result corresponds to a given set of parameters assumed in the above simulation, which involves that the $f(t)$ distributions and the relative contributions of AP-diff and CT-diff are fixed (i.e., only one $\lambda$ parameter for both components is let free in the fit). In this case, two AP-traps components with different mean release time $\tau$ were necessary to describe the data: a fast component with $\tau_{\text {fast }} \approx 25 \mathrm{~ns}$

\footnotetext{
${ }^{2}$ Afterpulses with short delay have smaller amplitudes as a consequence of the pixel recharging, and thus, they can be distinguished from dark counts and CT-diff pulses produced by fully recharged pixels.
} 


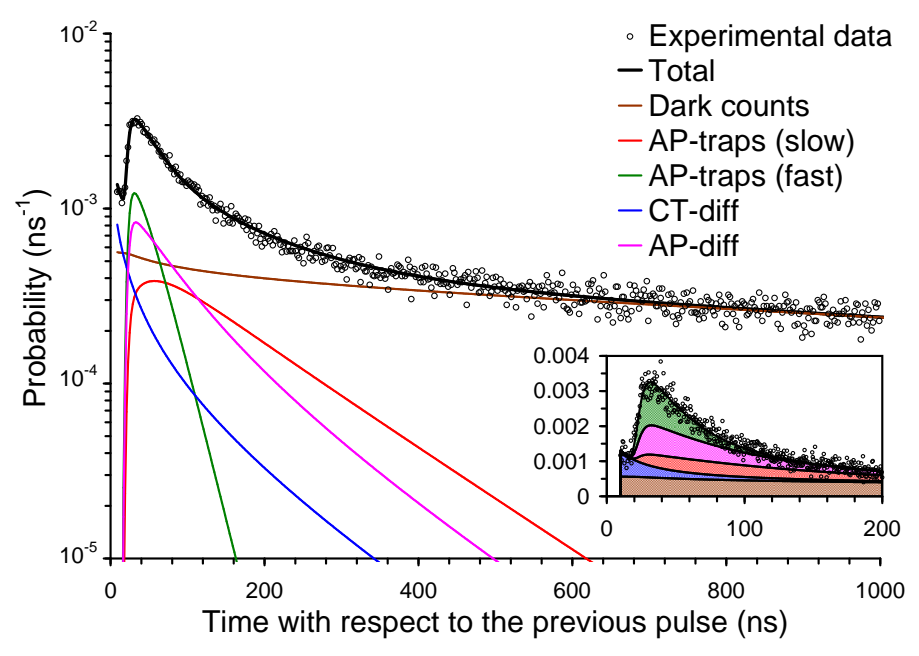

Figure 4: Fit of the model of afterpulsing and delayed crosstalk to the experimental time distribution of secondary pulses for the S10362-11-100C detector. The model predictions for each component of secondary pulses are shown. The accumulated contributions of the components at $t<200 \mathrm{~ns}$ are also shown in the inset (linear scale).

and a slow one with $\tau_{\text {slow }} \approx 150$ ns. On the other hand, when letting all the parameters related to AP-diff and CT-diff free, good quality fits were also obtained even omitting any AP-traps component. Similar results were obtained for the S10362-33-100C detector (not shown here). We are making the simulation more realistic in order to be more restrictive on the fitting parameters.

Even though the number of AP components has not been determined yet, the sum of the $\lambda$ parameters of all the AP components was found to be nearly independent of the assumptions made on the fit. The $\lambda$ parameter associated to the CT-diff was roughly fit independent as well, because it is basically determined by data at $t \lesssim 20 \mathrm{~ns}$, where AP contributions can be neglected. These $\lambda$ parameters, i.e., the one for the total contribution of afterpulsing and the one for CT-diff, are represented in figure 5 as a function of overvoltage, showing a pure quadratic behavior, as expected.3

\footnotetext{
${ }^{3}$ These results were obtained by fitting the $\lambda$ parameters, but using the $f(t)$ distributions determined in the fit shown in figure 4 at a given bias voltage. Appropriate corrections were applied to account for the overvoltage dependence of the detection threshold effects on the afterpulsing measurements.
} 


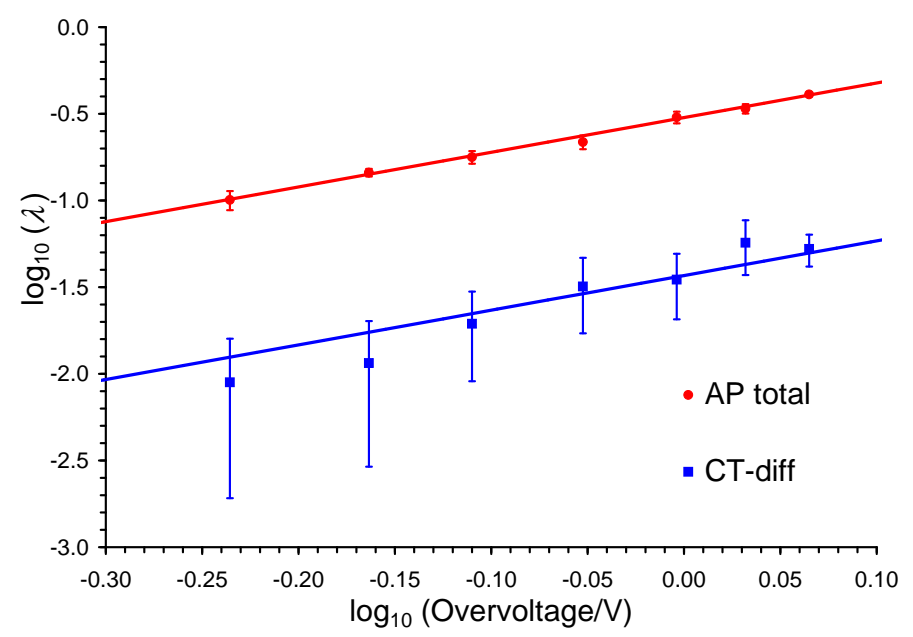

Figure 5: Average number of afterpulses (of any type) and pulses due to delayed crosstalk per primary avalanche as a function of overvoltage for the S10362-11-100C detector. The lines represent the best fits of quadratic functions of type $y=a \cdot x^{2}$. The uncertainties are statistical only.

\section{Conclusions}

We have developed an experimental method based on a waveform analysis to characterize the crosstalk and afterpulsing in SiPMs. Results were obtained for the S10362-11-100C and S10362-33-100C detectors from Hamamatsu at dark conditions and room temperature.

Measurements of optical crosstalk have been compared with an analytical model that we presented in a previous publication. Our data for both detectors are consistent with the hypothesis that optical crosstalk is only possible between adjacent pixels.

A statistical model of afterpulsing and delayed crosstalk has been constructed including a detailed parameterization of the time distributions of the different types of secondary pulses. Our preliminary results show a significant probability of afterpulsing in both detectors, although the characterization of its components is still under study. The delayed crosstalk was also found to be important, mostly at short time.

These studies are being extended to characterize other SiPMs and for a larger variety of conditions. We intend to apply our models to describe crosstalk and afterpulsing effects on particular experimental cases. 


\section{Acknowledgments}

This work was supported by MINECO (FPA2012-39489-C04-02) and CONSOLIDER CPAN CSD2007-42.

\section{References}

[1] L. Gallego, J. Rosado, F. Blanco and F. Arqueros, JINST 8 (2013) P05010.

[2] F. Nagy et al., Nucl. Instrum. Meth. A 759 (2014) 44.

[3] S. Vinogradov, Nucl. Instrum. Meth. A 695 (2012) 247.

[4] N. Otte, Nucl. Instrum. Meth. A 610 (2009) 105. 